\title{
Effects of centrifugal acceleration on the flows and segregation in vertical Bridgman crystal growth with steady ampoule rotation
}

\author{
C.W. Lan* \\ Department of Chemical Engineering, National Taiwan University, Taipei 10617, Taiwan, ROC
}

\begin{abstract}
The effects of centrifugal acceleration on the flows and segregation in vertical Bridgman crystal growth with steady ampoule rotation are investigated through numerical simulation. The numerical model is based on the Boussinesq approximation in a rotating frame, and the fluid flow, heat and mass transfer, and the growth interface are solved simultaneously by a robust finite-volume/Newton method. The growth of gallium-doped germanium $(\mathrm{GaGe})$ in the Grenoble furnace is adopted as an example. The calculated results at small Froude number $(\mathrm{Fr}<<1)$ are consistent with the previous prediction (Lan, J. Crystal growth 197 (1999) 983). However, at a high rotation speed or in reduced gravity, where the centrifugal acceleration becomes important $(\mathrm{Fr} \sim 1)$, the results are quite different due to the secondary flow induced. Since the direction of the induced flow is different from that of the buoyancy convection due to the concave interface, the flow damping is more effective than that due to the Coriolis force alone. More importantly, radial segregation can be reversed during the flow transition from one to the other. (C) 2001 Elsevier Science B.V. All rights reserved.
\end{abstract}

PACS: 44.25.+f; 47.27.Te; 81.10.Fq; 02.60.c6; 02.70Fj

Keywords: A1. Computer simulation; A1. Convection; A1. Interfaces; A1. Segregation; A2. Bridgman technique

\section{Introduction}

The control of heat flow, dopant segregation, and the growth interface is an important task in bulk crystal growth [1,2]. Using external forces to control crystal growth has been widely adopted [3-7]. Magnetic fields [4,5], the accelerated crucible

*Corresponding author. Tel.: + 886-2-2363-3917; fax: + 8862-2363-3917.

E-mail address: lan@ruby.che.ntu.edu.tw (C.W. Lan). rotation technique (ACRT)[6], and vibration (e.g., Ref. [7]) have been found useful. Crystal growth in a centrifuge (e.g., Refs. [8,9]) and reduced gravity [10] have also been proposed. However, the implementation of these techniques is usually costly and inconvenient. Recently, a novel approach using steady ampoule rotation for flow and segregation control was proposed by Lan [11]. With a high enough rotation speed, he found that the diffusion-controlled limit could be achieved for the vertical Bridgman (VB) configuration. Yeckel 
et al. [12] also adopted the same idea for the VB growth of $\mathrm{ZnCdTe}$, and also showed that the convection in the melt could be significantly suppressed by steady rotation. Lan [13] also obtained the scaling law of flow damping by the Coriolis force; the flow intensity decreases linearly with the rotation speed, or $\mathrm{Ta}^{-1 / 2}$. This scaling law applies to both concave and convex growth interfaces. A possible application of using steady rotation to reduce the unsteady and asymmetric segregation due to an arbitrary gravity orientation ( $g$-jitter) during crystal growth in space was also proposed by Lan [14]. Foster [15] also showed that, using an asymptotic analysis, the rotation only increases the radial segregation. Unfortunately, in the previous calculations [11-15], due to the use of a fixed frame and the Boussinesq approximation, the centrifugal acceleration was not considered, where the limiting case of the highspeed rotation is rigid-body rotation. However, at high-speed rotation or reduced gravity, the centrifugal force (acceleration) becomes important, which is perpendicular to axial temperature gradients and this can induce convection as well. Interestingly, the direction of the induced flow is different from that due to axial gravity for the concave interface. Therefore, in principle there will be a transition from one flow regime to the other, and this will alter radial dopant segregation. In a recent analysis by Wilcox et al. [16], they predicted the existence of a minimum convection for the concave interface through a simple thermal stability model, where the net acceleration of the body force is perpendicular to the isotherms. Unfortunately, this model does not consider the Coriolis force that damps the buoyancy flow both for the convex and concave interfaces.

In this paper, we will present a numerical model based on a rotating frame with the Boussniesq approximation so that the centrifugal acceleration can be considered in a consistent way. The benchmark system, the growth of gallium-doped germanium in the Grenoble furnace $[11,17,18]$, is considered. Some results showing very different flow and segregation behavior from the previous calculations are discussed and they may have interesting applications to crystal growth.

\section{Mathematical model and solution}

Based on the Boussinesq approximation, the governing equations in dimensionless form for the transport processes in the melt during crystal growth can be described by the conservation laws for the mass, momentum (in a rotating frame with a constant angular speed $\Omega$ about the crystal axis, i.e., $\boldsymbol{\Omega}=\boldsymbol{\Omega} \boldsymbol{e}_{z}$ ), energy, and dopant as follows:

$\nabla \cdot \boldsymbol{v}=0$,

$\frac{\partial \boldsymbol{v}}{\partial \tau}+\boldsymbol{v} \cdot \nabla \boldsymbol{v}=-\nabla P+\operatorname{Pr} \nabla^{2} \boldsymbol{v}+\boldsymbol{F}$,

$\frac{\partial T}{\partial \tau}+\boldsymbol{v} \cdot \nabla T=\nabla^{2} T$

$\frac{\partial C}{\partial \tau}+\boldsymbol{v} \cdot \nabla C=\frac{\operatorname{Pr}}{\mathrm{Sc}} \nabla^{2} C$,

where the body force term [19] can be written as:

$$
\begin{array}{rll}
\boldsymbol{F}=-\operatorname{Pr}_{T} \operatorname{Ra}_{T}\left(T-T_{\mathrm{m}}\right)\left[\boldsymbol{e}_{\mathrm{g}}+r \mathrm{Fr}_{\mathrm{r}}\right] & -\mathrm{T} a^{1 / 2} \operatorname{Pr} \boldsymbol{e}_{\mathrm{z}} \times \boldsymbol{v} . \\
\text { Gravitational } & \text { Centrifugal } & \text { Coriolis } \\
\text { acceleration } & \text { acceleration } & \text { force }
\end{array}
$$

In the above equations, $\boldsymbol{v}, P, T$, and $C$ are the dimensionless velocity, pressure, temperature and dopant concentration, respectively; $T_{\mathrm{m}}$ is the dimensionless melting temperature. Also, $\boldsymbol{e}_{\mathrm{g}}, \boldsymbol{e}_{\mathrm{r}}$, and $\boldsymbol{e}_{\mathrm{z}}$ are the unit vectors in the gravitational, radial and axial direction, respectively, and $r$ is the dimensionless radial distance. For the VB configuration here, $\boldsymbol{e}_{\mathrm{g}}=-\boldsymbol{e}_{\mathrm{z}}$. The associated dimensionless numbers as well as their physical meaning are summarized as the following:

$$
\begin{aligned}
& \operatorname{Ra}_{T}=\frac{\beta_{T} g \Delta T L^{3}}{v_{\mathrm{m}} \alpha_{\mathrm{m}}}=\frac{\text { Buoyancy force }}{\text { Viscous force }} \\
& \mathrm{Ta}=4 \Omega^{2} L^{4} / v_{\mathrm{m}}^{2}=\frac{\text { Coriolis force }}{\text { Viscous force }} \\
& \mathrm{Fr}=\Omega^{2} L / g=\frac{\text { Centrifugal acceleration }}{\text { Gravitational acceleration }},
\end{aligned}
$$

where $L$ is the domain length, $\Delta T$ the temperature difference between the hot and cold zones, $\mu_{\mathrm{m}}$ the melt viscosity, and $g$ the gravitational acceleration. In addition, $\operatorname{Pr}=v_{\mathrm{m}} / \alpha_{\mathrm{m}}$ is the Prandtl number and $\mathrm{Sc}=v_{\mathrm{m}} / D$ the Schmidt number, where $v_{\mathrm{m}}$ is the kinematic viscosity and $\alpha_{\mathrm{m}}$ and $D$ are the thermal and dopant diffusivities, respectively. For most of 
the dopants in the melt, $\mathrm{Sc} \gg 1$. Therefore, the dopant field, and thus the segregation, are dominated by the convection rather than by molecular diffusion.

In the above equations, the melt is assumed incompressible and the Boussinesq approximation is adopted. There is a controversy about the use of the fixed frame or the rotating frame in presenting the Boussinesq approximation. In the fixed frame approach, the density variation is put in the body force only, and the effect of centrifugal acceleration, which will generate pressure gradients in the radial direction, cannot be considered explicitly. As a result, at zero gravity, the steady ampoule rotation should result in a rigid-body rotation in the melt. However, in the rotating frame, the centrifugal force, i.e., $r$ Fre $\boldsymbol{e}_{\mathrm{r}}$, resembles a gravitational acceleration, and the density variation due to the temperature difference could be considered as shown above. Therefore, even at zero gravity, the thermal gradient in the axial direction, which is perpendicular to the centrifugal force, can induce flow. At normal gravity, the gravitational acceleration $g$ is usually much larger than the centrifugal acceleration $\left(r \Omega^{2}\right)$ at a moderate rotation speed, so that the Froude number $(\mathrm{Fr})$ is small and this effect can be neglected. However, when the centrifugal force is the dominant force, like in a high- $g$ centrifuge or reduced gravity environment, the rigid-body rotation solution is not valid any more, and the Sweet-Eddington flow [20] may be induced.

With suitable boundary conditions [11], the above governing equations can be solved numerically by an efficient finite-volume/Newton method [11,18] with second-order accuracy. Detailed numerical implementation can be found elsewhere.

\section{Results and discussion}

For VB crystal growth, due to its thermally stable configuration and thus weaker buoyancy flow, the flow damping by ampoule rotation is usually effective as proposed by Lan [11]. For comparison purposes, we again take the growth of gallium-doped germanium (GaGe) in the Grenoble furnace as an example. This problem, without rotation, was also investigated by Adornato and Brown [17]. Extensive benchmark numerical comparisons were also performed [18] and therefore it serves as a good candidate for the theoretical study here. The typical flow damping by the steady ampoule rotation is shown in Fig. 1 for $\mathrm{R} a_{T}=2.489 \times 10^{8} \quad$ (at normal gravity) and $2.489 \times 10^{6}$ (at $0.01 \mathrm{~g}$ ). Some typical flow structures are illustrated in Fig. 2. As shown in Fig. 1, at a lower rotation speed (or Ta), the flow intensity $\left(\left|\Psi_{\max , \min }\right|\right)$ for the major flow cell (in front of the interface) is suppressed significantly by rotation, and the scaling law (for $\mathrm{Fr}=0$ ) follows nicely with the boundary layer approximation, i.e., $\left|\Psi_{\max }\right| \sim \mathrm{Ta}^{-1 / 2}$. However, as the rotation speed increases, the discrepancy of the two models $(\mathrm{Fr}=0$ and $>0)$ increases. At $100 \mathrm{RPM}, r \mathrm{Fr}$ (or $R_{\mathrm{c}} \Omega^{2} / g$ ) is about 0.056 for $1 \mathrm{~g}$ and 5.6 for $0.01 \mathrm{~g}$ (about 0.223 at $20 \mathrm{RPM}$ ), where $R_{\mathrm{c}}$ is the crystal radius being $0.5 \mathrm{~cm}$ here. The flow intensity of the secondary cell, which is induced by the centrifugal acceleration, is also plotted. As shown, the

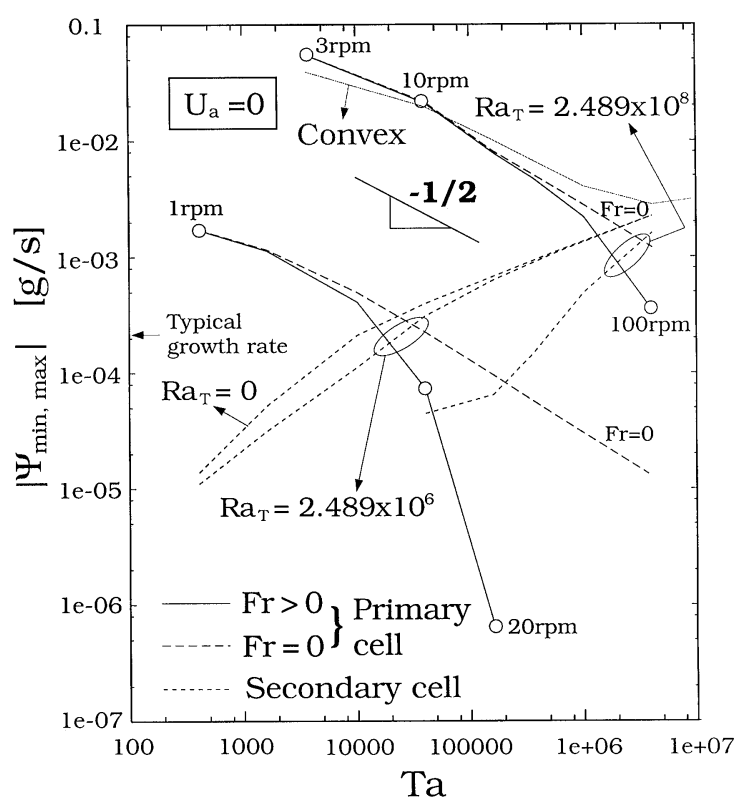

Fig. 1. Variation of flow intensity with the increasing Taylor number for $\mathrm{Ra}_{T}=2.489 \times 10^{6}(0.01 \mathrm{~g})$ and $2.489 \times 10^{8}$ (normal gravity); $U_{\mathrm{a}}=0$. The primary cell is the flow in front of the interface. The result for the convex interface (by exchanging the crystal and melt thermal conductivities) is also added at the top. 


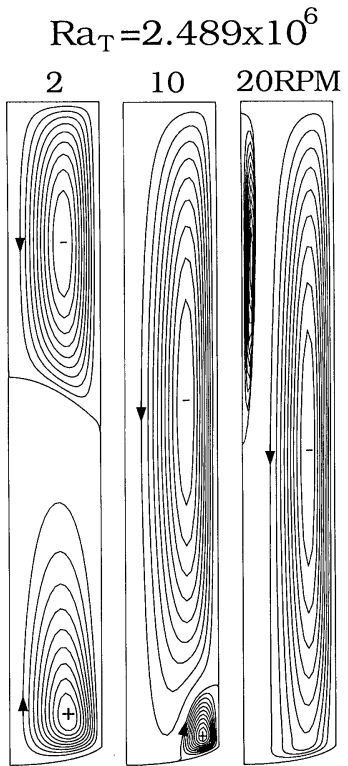

(a)

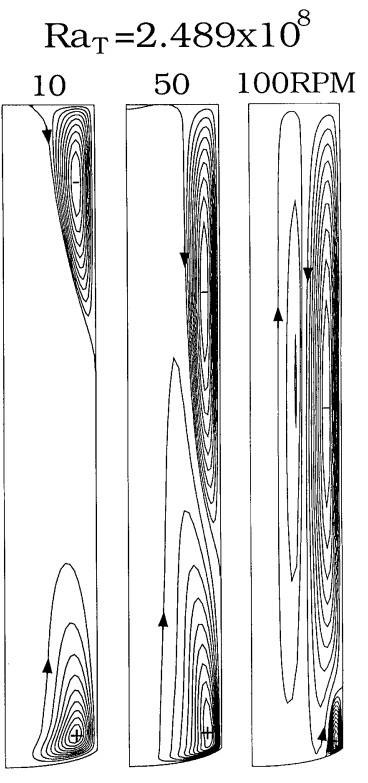

(b)
Fig. 2. Some flow structures selected from Fig. 1; (a) $\mathrm{Ra}_{T}=2.489 \times 10^{6}(0.01 \mathrm{~g})$; (b) $2.489 \times 10^{8}$ (normal gravity); For (a), $\Psi_{\min }=-3.168 \times 10^{-5} \mathrm{~g} / \mathrm{s}, \Psi_{\max }=1.127 \times 10^{-3} \mathrm{~g} / \mathrm{s}$, for 2 $\mathrm{RPM} ; \Psi_{\min }=-3.104 \times 10^{-4} \mathrm{~g} / \mathrm{s}, \quad \Psi_{\max }=7.282 \times 10^{-5} \mathrm{~g} / \mathrm{s}$, for $10 \mathrm{RPM} ; \Psi_{\min }=-6.304 \times 10^{-4} \mathrm{~g} / \mathrm{s}, \Psi_{\max }=6.728 \times 10^{-7} \mathrm{~g} / \mathrm{s}$ for $20 \mathrm{RPM}$; For (b) $\Psi_{\min }=-4.466 \times 10^{-5} \mathrm{~g} / \mathrm{s}, \Psi_{\max }=0.0211 \mathrm{~g} / \mathrm{s}$, for $10 \mathrm{RPM} ; \Psi_{\min }=-4.899 \times 10^{-4} \mathrm{~g} / \mathrm{s}, \Psi_{\max }=2.187 \times 10^{-3} \mathrm{~g} / \mathrm{s}$, for $50 \mathrm{RPM} ; \Psi_{\min }=-1.598 \times 10^{-3} \mathrm{~g} / \mathrm{s}, \Psi_{\max }=3.447 \times 10^{-4} \mathrm{~g} / \mathrm{s}$ for 100 RPM.

centrifugal force begins to dominate at high $\mathrm{Ta}$ number, and its flow intensity approaches to the limit of $\operatorname{Ra}_{T}=0$, which is the basic flow due to the centrifugal force alone. Because the flow direction of the secondary cell (due to axial thermal gradients) induced by the centrifugal force is different from that of the primary cell (due to radial thermal gradients), as illustrated by Fig. 2, the flow damping of the major cell becomes much more effective than that by the Coriolis force alone, as shown in Fig. 1. Finally, further increasing the rotation speed, the secondary cell prevails and the major cell in front of the growth interface disappears. Clearly, the flow transition will lead to the minimum convection near the interface, and the segregation seems to approach the diffusion limit due to the concave interface. Meanwhile, if the rotation speed is slightly higher, a minimum

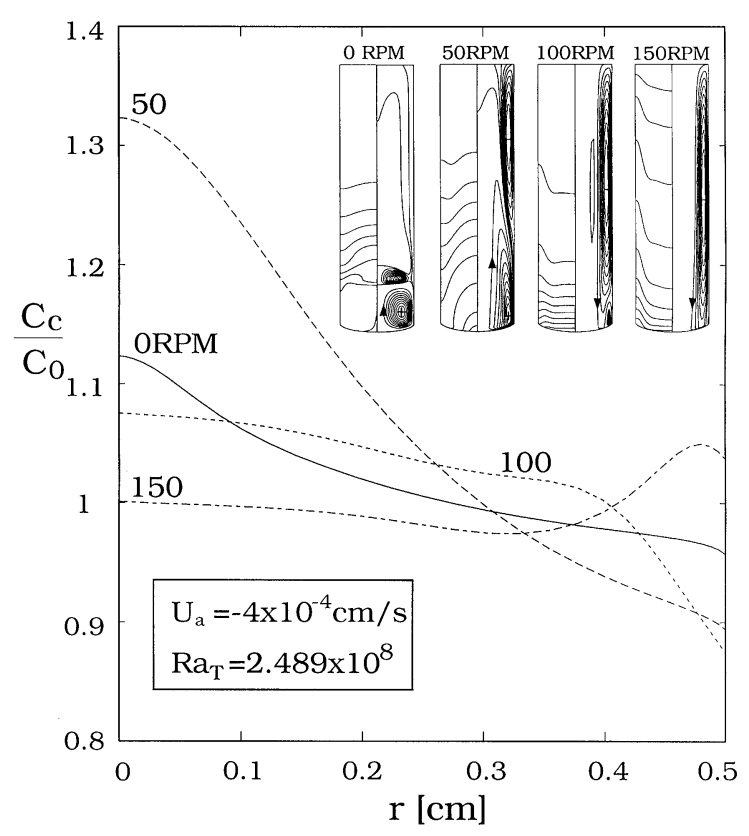

Fig. 3. Effects of rotation on radial dopant segregation for $\mathrm{Ra}_{T}=2.489 \times 10^{8}$. Some flow and dopant fields are also illustrated, where $\Psi_{\min }=-0.00136 \mathrm{~g} / \mathrm{s}, \quad \Psi_{\max }=0.05576 \mathrm{~g} / \mathrm{s}$, $C_{\min }=1.0595$, and $C_{\max }=12.9125$ for $0 \mathrm{RPM}$; $\Psi_{\min }=$ $-6.249 \times 10^{-4} \mathrm{~g} / \mathrm{s}, \quad \Psi_{\max }=0.00221 \mathrm{~g} / \mathrm{s}, \quad C_{\min }=1.188, \quad$ and $C_{\max }=15.21$ for $50 \mathrm{RPM} ; \Psi_{\min }=-1.741 \times 10^{-3} \mathrm{~g} / \mathrm{s}, \quad \Psi_{\max }=$ $1.188 \times 10^{-4} \mathrm{~g} / \mathrm{s}, \quad C_{\min }=1.3191, \quad$ and $\quad C_{\max }=12.359$ for $100 \mathrm{RPM} ; \quad \Psi_{\min }=-2.678 \times 10^{-3} \mathrm{~g} / \mathrm{s}, \quad \Psi_{\max }=0 \mathrm{~g} / \mathrm{s}, \quad C_{\min }=$ 2.4556 , and $C_{\max }=12.067$ for 150 RPM.

segregation can be expected, which will be illustrated shortly. Furthermore, the rotation speed for the minimum convection predicted by Wilcox et al. [16] is overestimated being about $50 \%$ to $100 \%$ higher.

If the growth rate is considered by setting the ampoule pulling speed $U_{\mathrm{a}}$ as $-4 \times 10^{-4} \mathrm{~cm} / \mathrm{s}$, the dopant segregation can be investigated. Some pseudo-steady state calculations are shown in Fig. 3 for the radial segregation; typical flow and dopant fields are included for comparison. As shown, as predicted by Lan [11], a diffusionlimited regime, such as the case for 100 RPM, can be approached. However, due to the centrifugal acceleration, there is some distortion of the dopant field along the ampoule wall. Further increasing the rotation speed will increase dopant mixing in the bulk melt. Nevertheless, near the growth 


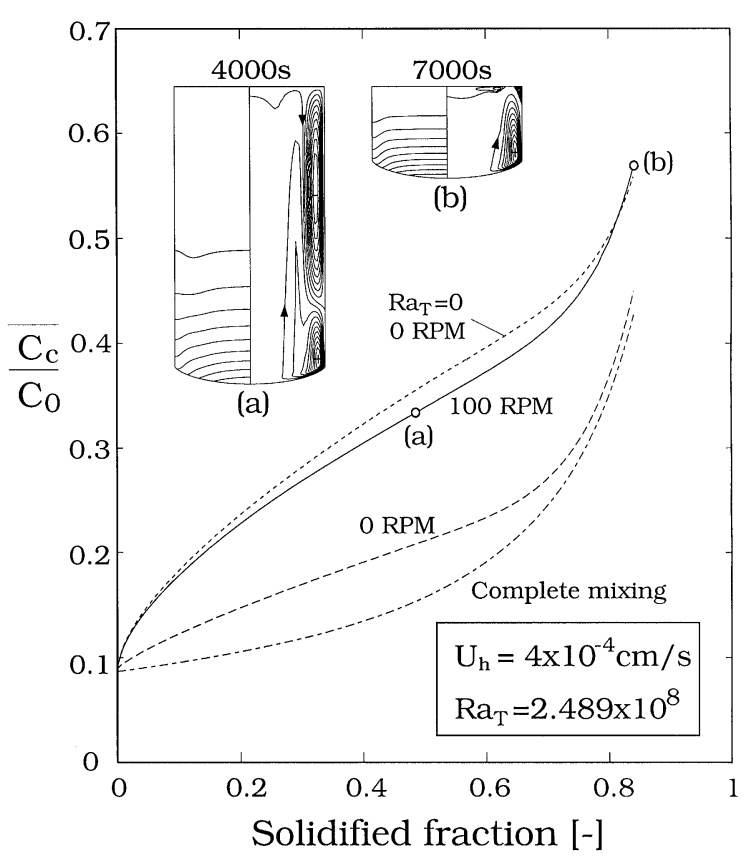

Fig. 4. Axial dopant segregation in the grown crystal; the flow and dopant fields for 100 RPM at 4000 s (about $46 \%$ solidified) and $7000 \mathrm{~s}$ (about $84 \%$ solidified) are included, where $\Psi_{\min }=-5.331 \times 10^{-4} \mathrm{~g} / \mathrm{s}, \quad \Psi_{\max }=5.217 \times 10^{-4} \mathrm{~g} / \mathrm{s}, \quad C_{\min }=$ 1.0850 , and $C_{\max }=4.417$ at $4000 \mathrm{~s}$ and $\Psi_{\min }=-1.145 \times$ $10^{-5} \mathrm{~g} / \mathrm{s}, \Psi_{\max }=4.990 \times 10^{-4} \mathrm{~g} / \mathrm{s}, C_{\min }=3.94$, and $C_{\max }=7.384$ at $7000 \mathrm{~s}$. The initial condition is the same as Fig. 2(b) at $100 \mathrm{RPM}$ and $U_{\mathrm{h}}$ is the heater moving speed.

front, the dopant transport is more or less diffusion limited. From Fig. 3, it is also clear that the radial dopant segregation is reversed between 100 and 150 RPM due to changing flow direction in front of the interface (the exchange of two flow cells). Therefore, as shown in Fig. 3 there is a minimum radial segregation and it occurs right after the flow transition. In other words, the use of ampoule rotation (say 150 RPM) for flow damping and further segregation control seems to be feasible. Although the conclusion is similar to the one by Lan [11], the minimum radial segregation was not predicted in the previous calculations, where the rigid-body rotation was the high-speed limit.

A final example is illustrated by dynamic calculations. Fig. 4 shows the axial dopant segregation in the grown crystal (averaged over the cross section) for 100 RPM. The cases of no rotation, diffusion-limited, and complete-mixing are also included for comparison. Again, as shown, with 100 RPM rotation, the axial segregation is quite close to the diffusion-limited one. On the other hand, without rotation, the local dopant mixing due to the buoyancy flow leads to the axial segregation close to the complete-mixing limit. To further control the radial segregation, a higher rotation speed can be used as discussed. From the calculated results, one may expect to achieve a uniform axial segregation through a decreasing rotation speed. In other words, at the beginning of growth, one can use faster rotation, which leads to a dopant boundary layer in front of the interface to build up quickly. As the growth proceeds, the rotation speed can be gradually reduced. A similar idea was also adopted by Ozawa et al. [21] by using a stepwise reduction of a magnetic field.

\section{Conclusions}

In this paper, we revise previous calculations by including the centrifugal acceleration using a rotating frame. The flow damping and segregation control by steady ampoule rotation are discussed. At a low rotation speed or small Froude number, the flow intensity decreases with the increasing rotation speed and the rigid-body rotation is the high-speed limit. With the centrifugal acceleration, the axial thermal gradient can induce buoyancy flow due to the radial body force, and its flow direction is different from that of the buoyancy flow induced by the concave growth front. As a result, the flow damping of the primary cell by rotation becomes even more effective than that by the Coriolis force alone. The radial segregation can be reversed right after the flow transition. With such a flow characteristics, in principle it is expected that the segregation control by steady ampoule rotation, both radial and axial, can be achieved. Furthermore, because both the Coriolis and centrifugal forces are axisymmetric, the use of ampoule rotation should be helpful in reducing asymmetric heat flow and segregation due to imperfect growth conditions. 


\section{References}

[1] R.A. Brown, AIChE J. 34 (1989) 881.

[2] G. Müller, A. Ostrogorsky, in: D.T.J. Hurle (Ed.), Handbook of Crystal Growth 2b: Growth Mechanisms and Dynamics, North-Holland, Amsterdam, 1994.

[3] H.P. Utech, M.C. Flemming, J. Appl. Phys. 37 (1966) 2021.

[4] K.M. Kim, J. Electrochem. Soc. 132 (1982) 427.

[5] D.H. Kim, P.M. Adornato, R.A. Brown, J. Crystal Growth 89 (1988) 339.

[6] H.J. Scheel, J. Crystal Growth 13/14 (1971) 560.

[7] C.W. Lan, Int. J. Heat Mass Transfer 43 (2000) 1987.

[8] W.A. Arnold, W.R. Wilcox, A. Frederick Carlson, L.L. Chait, Regel, J. Crystal Growth 119 (1992) 24.

[9] G. Müller, G. Neumann, W. Webber, J. Crystal Growth 119 (1992) 8.

[10] A.F. Witt, H.C. Gatos, M. Lichtensteiger, M.C. Lavine, C.J. Herman, J. Electrochem. Soc. 122 (1975) 276.
[11] C.W. Lan, J. Crystal Growth 197 (1999) 983.

[12] A. Yeckel, F.P. Doty, J.J. Derby, J. Crystal Growth 203 (1999) 87.

[13] C.W. Lan, J. Chin. Inst. Chem. Eng. 31 (2000) 465.

[14] C.W. Lan, M.C. Liang, J.H. Chian, J. Crystal Growth 212 (2000) 340.

[15] M.R. Foster, J. Fluid Mech. 409 (2000) 185.

[16] W.R. Wilcox, L.L. Regel, W.A. Arnold, J. Crystal Growth 187 (1998) 543.

[17] P.M. Adornato, R.A. Brown, J. Crystal Growth 80 (1987) 155.

[18] C.W. Lan, F.C. Chen, Comput. Meth. Appl. Mech. Eng. 131 (1996) 191.

[19] J. Friedrich, J. Baumgartl, H.-J. Leister, G. Müller, J. Crystal Growth 167 (1996) 45.

[20] G. Buzyna, G. Veronis, J. Fluid Mech. 50 (1971) 579.

[21] S. Ozawa, T. Kimura, J. Kobayashi, T. Fukuda, Appl. Phys. Lett. 50 (1987) 329. 